\title{
Developing a robust design basis for critical infrastructures against extreme external floods
}

\author{
J.-U. Klügel \\ NPP Goesgen-Daeniken, Switzerland
}

\begin{abstract}
Extreme floods represent a major threat to human civilization. Insurance losses caused by floods have a large contribution to risk from natural events. Big river floods in Europe (Germany 2002, Middle Europe 2013) have challenged the emergency preparedness of governments and caused regional critical situations including human losses. The occurrence of extreme external floods has frequently surprised the designers and operators of critical infrastructures and lifelines. After the Fukushima disaster nuclear authorities recognized that extreme floods may have significant consequences on the safety of nuclear power plants. Therefore both IAEA as well as WENRA required the development of robust methods of hazard analysis for new plants as well as for the safety review of existing plants to avoid cliff edge effects. The paper presents the application of a recently developed method of hazard assessment for extreme natural events for the case of extreme external floods. The method allows for the consideration of unexpected extreme events ("black swan" events) of natural origin delivering the basis of a robust design of safety features. The hazard assessment method is making use of some general properties of heavy tail distributions and theory of records. The new method is combined with traditional statistical hazard assessment methods in a procedure for the development of a robust design basis for extreme river flood protection for critical infrastructures. The application of the method is demonstrated on the example of developing the design basis for a nuclear power plant against extreme external floods. The hazard assessment results are compared with the results of hazard assessment using conventional probabilistic and modelling hazard analysis methods. The risk and design implications are discussed.

Keywords: Black Swan Theory, extreme floods, hazard assessment, critical infrastructures.
\end{abstract}




\section{Introduction}

Extreme floods represent a major threat to human civilization. Insurance losses caused by floods have a large contribution to risk from natural events. Big river floods in Europe (Germany 2002, Middle Europe 2013) have challenged the emergency preparedness of governments and caused regional critical situations including human losses. The occurrence of extreme external floods has frequently surprised the designers and operators of critical infrastructures and lifelines. A robust design of critical infrastructures against extreme floods like nuclear power plants is of special importance. After the Fukushima disaster nuclear authorities recognized the high risk potential of natural hazards including floods. Therefore both IAEA (International Atomic Energy Agency) [1] as well as WENRA (Western Europe Nuclear Regulatory Association) [2] required the development of robust methods of hazard analysis for new plants as well as for the safety review of existing plants to avoid cliff edge effects. Risk-informed approaches like that discussed in INSAG [3] focus on a holistic approach to safety decision making ensuring the robustness of decisions. The risk-informed framework outlined by INSAG [3] endorses the use of different analysis methods. They complement each other and their use leads to improved and deeper safety insights. Here a new method for extreme flood hazard analysis is proposed with the main focus given to extreme river floods. In conjunction with established methods this new method can be applied to develop a robust design basis for extreme floods for critical infrastructures without leading to an undue conservatism.

\section{Short overview on river flood hazard assessment methods}

In most European countries a probabilistic approach for extreme flood hazard assessment is in use, which may be complemented by a modelling approach. The DWA guideline DWA-M 552 [4] gives an overview on different statistical methods for the assessment of the probability of extreme floods that are in practical use. The guideline distinguishes between:

- Statistical methods based on local flood data;

- Methods that are based on the evaluation of large historical floods;

- Modelling methods for relevant critical flow areas;

- Statistical methods that are based on regional data.

In general it is well recognized that statistical approaches to flood analysis have limitations due to lack of data or due to human interference that may have changed the boundary conditions for the occurrence of extreme floods. In [4] the benefits and drawbacks of each of the methods are described. To achieve an improved performance of the hazard assessment it is recommended to combine the different approaches for the assessment. Nevertheless, this is not always possible due to lack of information or it may require expert judgment which is subjective.

For some critical infrastructures like nuclear power plants (NPPs) more specific guidelines exist that shall ensure a robust hazard assessment. The latter 
is achieved by using an intentionally conservative parametric heavy-tail distribution as for example in KTA [5].

In Klügel [6] traditional statistical methods (based on local statistics) are used for the probabilistic risk assessment for extreme river floods for a nuclear power plant. The risk assessment results are strongly dependent on the accuracy of the hazard assessment and the uncertainty of calculated risk metrics may reach several orders of magnitude.

\section{Procedure for the development of the design basis for critical infrastructures for extreme river flood protection}

The procedure developed represents a specific form of aggregation of the results from different hazard assessment methods combining traditional approaches with a newly developed distribution free method which is based on "Black Swan Theory".

\subsection{General flood hazard assessment procedure: overview}

The objective of the hazard assessment procedure consists in the definition of a design basis flood and a review level beyond design basis flood. The purpose of the latter is to perform "what-if-analyses" to exclude cliff-edge effects by considering appropriate mitigating measures in the design of critical infrastructures.

The procedure for the development of the design basis of a critical infrastructure for extreme river floods consists of the following principal steps:

1) Collection and evaluation of historical data of river floods in the near site vicinity of the site (local statistics).

2) Identification of anthropomorphic modifications to river beds and canals which may have an impact of flood conditions near the site.

3) Definition of a homogenized data series for flood data which is applicable for the actual site conditions, for example on the basis of statistical tests for i.i.d conditions.

4) Development of probabilistic models for extreme water flow conditions (standard statistical methods) and selecting the most appropriate model (see for example, Klügel [6]) or weighing them in a logic tree (if epistemic uncertainty associated with the use of different probabilistic models has to be considered according to national standards).

5) Selection of a design basis flood and a beyond design basis extreme flood using decision criteria set in national standards (e.g. for nuclear power plants frequently the mean hazard with a probability of exceedance of $10^{-4}$ is used for design, and an additional safety factor of 1.5 is used to define a beyond design basis flood).

6) Prediction of the next record flood expected during the lifetime of the infrastructure (design basis flood) and the second in the series of records flood (beyond design basis flood) according to the method proposed in section 3.2 ("Black Swan Theory"). 
7) Check of the physical feasibility of the flood scenarios developed in step 6) based on a review of rain and flood data and their recurrence intervals and of hydrological flow boundary conditions (e.g. extreme floods may alter the river bed conditions).

8) Definition of a design basis river flood that is at least higher or equal to the predicted record event from step 6). For example, it may be reasonable to select the maximum flood from steps 5) and 6) of the procedure. Additionally an extreme beyond design basis flood should be developed. The smaller of the beyond design basis floods from step 5) (if it is larger than the design basis flood from 6)) and step 6) is recommended to be used to avoid an overly conservatism.

9) Development of hydrographs for the design basis flood and the extreme beyond design flood either based on historical data, rainfall and hydrological simulation or using a synthetic hydrograph.

10) Documentation and dissemination of the results of the hazard assessment for application in the design of the critical infrastructure.

\subsection{Hazard assessment based on "Black Swan Theory"}

The objective of the new hazard assessment method consists in the prediction of an "upper limit" extreme flood that surpasses all previous historical observations still being possible under the environmental conditions characteristic for a critical infrastructure of limited lifetime. Events that are highly improbable but may have a significant impact are commonly denoted as "black swan" events in reference to the popular book of Nassim Taleb [7]. So the key point of the method consists in the prediction of "Black Swan" events. The method proposed makes use of some general properties of the mathematical theory of records and of the theory of heavy-tail distributions. Mathematically, it represents a distribution free extension of extreme value theory. After the financial crisis of 2008 it became popular to denote advanced applications of extreme value theory as "Black Swan Theory" as for example in Klügel [8], and [9]. The method assumes that the recurrence of extreme natural events follows a stochastic process which is caused by the same, but unknown mechanism (corresponds mathematically to the i.i.d assumption for a sequence of random variables (rvs))). Furthermore it is assumed that the magnitude of the extreme event is following a heavy-tail distribution. The latter is a direct consequence of the Fisher-Tippett theorem; see Embrechts [10] under the i.i.d. assumption. Furthermore, it is frequently believed that the occurrence of extreme natural hazards can be explained as a phenomenon of self-organized criticality. The mathematical description of self-organized criticality directly leads to extreme value theory and heavy-tail distributions (Woo [11]).

The validity of the assumptions can be expanded mathematically to the more general case of weak asymptotic convergence to a Homogeneous Poisson Process (HPP) (e.g. for Poissonian Random Measures (PRM)). This asymptotic convergence is proven for point processes of exceedances over a threshold using the Kallenberg theorem (Embrechts [10]). Note that any new record represents an exceedance over a threshold. Therefore, the mathematical prerequisites for the 
applicability of the assumptions of the method are less restrictive than the standard assumptions in probabilistic risk assessments (see for example section 2.2.2 in NRC [12]).

\subsubsection{General properties of the mathematical theory of records and of heavy-tail distributions}

Here we summarize some general properties of the theory of records and of heavy-tail distributions that are used in the method proposed (see for comparison [9]). A detailed overview on the mathematical basis of the theory of records and of heavy- tail distributions can be found in Nevzorov [13], Cook and Nieboor [14] and in the mathematical text books Embrechts [10] and Resnick [15].

1) The expected number of records (denoted as $N_{n}$ ) in a sequence of $n$ observations is given by:

$$
E\left(N_{n}\right)=\sum_{j=1}^{n} \frac{1}{j}
$$

2) The ratio between the largest and the second largest record in a record counting process for a heavy tailed distribution converges asymptotically to a factor of 2 . The probability that the next record value will exceed the second largest (the previously observed) record value by a factor of 2 or more is equal to:

$$
\frac{2}{n}
$$

The first property (eqn (1)) defines the expected time of occurrence of the next record value. Regarding extreme natural events it defines the waiting time till the next extreme event exceeding all previous observations is expected to occur. From eqn (1) it also follows that the longer the historical record of observations is the more records will be observed, while the waiting time till the next record is increasing (by a factor of $e$ for the median of the waiting time distribution, Embrechts [10]). The second property defines the asymptotic behaviour of the magnitude of the event. Of course statistically the next record event may have any magnitude exceeding all previously observed values but the probability that it will exceed the previous record by a factor of 2 or more converges asymptotically to zero with an increasing number of observations. From both properties it follows,

1) that in case of a long historical record and for a critical infrastructure of limited lifetime the occurrence of a new record that has a high impact and comes as a surprise (this is the definition of a "Black Swan" event) is quite unlikely; and

2) that the magnitude of the next record event will most likely not exceed the previous record value by more than a factor of 2 .

\subsubsection{Definition of "Black Swan" events}

The method proposed is based on the prediction of the largest extreme event to be expected during the residual or planned lifetime of a critical infrastructure. Certainly this event shall have a larger magnitude then previously observed 
events to incorporate some safety factor into the design of the critical infrastructure. By definition such an event will be a record in the mathematical sense. Using the asymptotic properties of extremes with characteristics of a heavy-tail distribution (see eqn (2)) it is reasonable to assume that the magnitude of this next extreme record event exceeds the previously observed record value by a factor of 2 . The exceedance of the magnitude of all previous observations by a factor 2 certainly will be a surprise for external observers. On the other hand such an event will be very rare. This can be illustrated by an example. Assuming the availability of an observational data set of 100 observations, it follows from eqn (2) that the probability that the next record will exceed the previous one - by at least a factor of $2-$ is just $2 \%$ (it may be weaker). Furthermore, the expected waiting time till this record event will take place by definition shall exceed the lifetime of the critical infrastructure. According to eqn (1) the expected number of records for 100 observations is approximately five. The expected waiting time till the next record value from eqn (1) is 126 years. Typically this is significantly longer than the usual lifetime of a critical infrastructure (e.g. for nuclear power plants 40-60 years, for buildings maximum 100 years). Therefore the combination of the two properties given by eqn (1) and eqn (2) ensures that the predicted record event is both very strong and very rare. These properties correspond to the usual definition of "Black swan" events (e.g. highly improbable, but of large impact).

\subsubsection{Hazard parameter}

To characterize the magnitude of the "Black Swan" event it is necessary to identify the hazard parameter to which the "multiplication by a factor of 2" rule (property 2) in 3.2.1, eqn (2)) shall be applied. In Klügel $[8,9]$ it is discussed in detail that the intrinsic energy of the hazardous event is the most appropriate parameter for the characterization of the magnitude of the extreme event. Therefore for extreme river floods the kinetic energy of the flow is used as hazard parameter.

\subsubsection{Design basis and beyond design basis floods}

The new method can be used to develop a design basis river flood as well as a beyond design basis river flood. The design basis river flood corresponds to the predicted next "extreme" record river flow, while the beyond design basis river flood corresponds to the predicted second in the sequence of records river floods.

\section{Practical application for a nuclear power plant}

\subsection{Local conditions}

According to Swiss national regulations the licensees of nuclear power plants have to perform periodic safety reviews including a review and update of natural hazards assessments. The new procedure was applied for the post-Fukushima safety review of NPP Goesgen as well as for the next plant specific periodic safety review. 


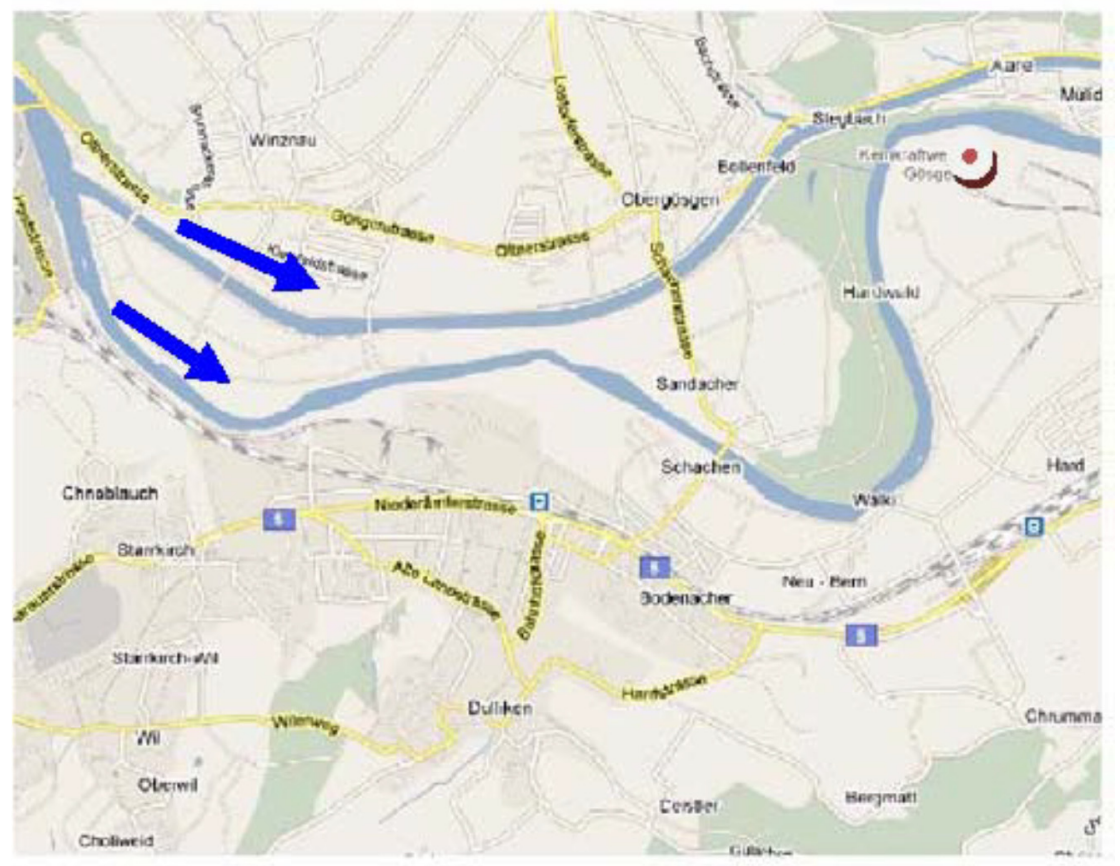

Figure 1: Location of NPP Goesgen at the river Aare.

Figure 1 shows the location of NPP Goesgen downstream of the watershed between the river bed of the Aare and the upper channel of the close by hydropower station Goesgen. The watershed is formed by the weir of Winznau. For the estimation of floods it is necessary to model the water flow into the Aare which is depending on the flow distribution between the Aare and the upper channel of the hydropower station. Therefore the operation of the hydropower station affects the flow conditions in the Aare. For extreme floods it can be assumed conservatively that the flow through the upper channel is jammed to a large extent by clogging material transported with the river flow. The next local measurement station of the Aare is in Murgenthal. There is a well-established correlation between the measurement data at the station Murgenthal and the river flow arriving at the weir in Winznau:

$$
H Q_{\text {Winznau }}=1.073 H Q_{\text {Murgenthal }}
$$

Here HQ denotes the river flow in $\mathrm{m}^{3} / \mathrm{s}$. Therefore it is possible to apply standard statistical methods to predict extreme river flows in the Aare close to the NPP Goesgen power station based on local measurement data.

\subsection{Observation data}

Measurement data at the station Murgenthal is available since 1916. Nevertheless in the early seventies large correction measures of the flow path of 
the Aare were completed that may affect the local conditions at the Goesgen site. As a consequence a set of statistical tests was performed to check the acceptability of the i.i.d assumption for the data set available. The i.i.d. assumption could not be rejected. This allows using the whole data set for the analysis.

Additionally, a review of reported historical river floods was performed. The scope of review reached back to the 13th century (1268). The river bed of the Aare was completely changed in the second half of the 19th century. Therefore, extrapolations from historical floods to the actual flow conditions were based on a modelling approach. The analysis included an evaluation of the influence of regional precipitation on the river flow in the Aare. This allowed developing a widely homogenized data set for statistical analysis reaching back to the 13th century. This extended data set was applied for statistical analysis.

\subsection{Results derived by parametric statistical analysis}

Different statistical methods were applied to the expanded dataset. For the data fit the software tools ModelRisk ${ }^{\circledR}$ [16] and MATLAB ${ }^{\circledR}$ were used. The results obtained for different return levels (in terms of probability of exceedance) and different parametric models are shown in Table 1. The results (several other models were tested, too) show considerable differences. The statistically best performing model (using assessment criteria from information theory [16]) was the model of Gamma distribution. According to the procedure proposed the design basis flood (for a probability of exceedance of $10^{-4}$ for NPP Goesgen) corresponds to a river flow of $1582.2 \mathrm{~m}^{3} / \mathrm{s}$. The associated beyond design basis flood is derived by multiplication with a safety factor of 1.5 and makes up a flow of $2373.3 \mathrm{~m}^{3} / \mathrm{s}$.

Table 1: Extreme river flows near the Goesgen NPP site (parametric analysis).

\begin{tabular}{|c|c|c|c|}
\hline $\begin{array}{c}\text { Probability of } \\
\text { exceedance }\end{array}$ & $\begin{array}{c}\text { Gamma distribution, } \\
\text { flow }\left[\mathrm{m}^{3} / \mathrm{s}\right]\end{array}$ & Gumbel distribution & $\begin{array}{c}\text { Generalised } \\
\text { Extreme value } \\
(\text { GEV) distribution }\end{array}$ \\
\hline $10^{-2}$ & 1233.1 & 1280.9 & 1177.7 \\
\hline $10^{-3}$ & 1417.8 & 1461.5 & 1347.3 \\
\hline $10^{-4}$ & 1582.2 & 1667.6 & 1485.0 \\
\hline $10^{-5}$ & 1734.3 & 1902.8 & 1596.7 \\
\hline $10^{-6}$ & 1777.9 & 2171.1 & 1687.4 \\
\hline
\end{tabular}

\subsection{Results derived by "Black Swan Theory"}

The new distribution free approach to hazard assessment is applied to the measured local river flow data (without extension to historical data).

Since the start of flow measurements a total of 6 record values of annual maximal river flow have been observed. These records are shown in Table 2.

This is in good agreement with the expected number of records of 5.2 (that means between 5 and 6 record events) calculated from eqn (1). According to the assessment method the next predicted record value results is obtained by 
Table 2: $\quad$ Record river flow observations since the beginning of measurements in 1916.

\begin{tabular}{|c|c|}
\hline Date $(\mathrm{y} / \mathrm{m} / \mathrm{d})$ & Flow $\left[\mathrm{m}^{3} / \mathrm{s}\right]$ \\
\hline 1916.12 .27 & 858.4 \\
\hline 1918.12 .24 & 940.0 \\
\hline 1944.11 .24 & 987.2 \\
\hline 1972.11 .23 & 1051.5 \\
\hline 1981.03 .12 & 1094.5 \\
\hline 2007.08 .09 & 1352.2 \\
\hline
\end{tabular}

doubling the kinetic energy of the river flow. For a constant flow area this is equivalent to the multiplication of the last record flow value by $\sqrt{2}$. Therefore, we obtain for the design basis flood a value of $1912.3 \mathrm{~m}^{3} / \mathrm{s}$. This is a sufficiently rare event with an expected waiting time lasting till 2077. This time is far beyond the planned lifetime of NPP Goesgen. The more extreme beyond design basis flood proposed for the evaluation of possible "cliff edges" is $2704.4 \mathrm{~m}^{3} / \mathrm{s}$. The expected waiting time would last till 2198. Due to the limited residual lifetime of NPP Goesgen it can be excluded that such an event will be observed before the final decommissioning of the plant.

\subsection{Design basis and beyond design basis flood according to the procedure}

Combining the results from the different analyses (step 8) of the procedure) the final design basis flood corresponds to a river flow of $1912.3 \mathrm{~m}^{3} / \mathrm{s}$.

The beyond design basis scenario according to the procedure results in a river flow of approximately $2375 \mathrm{~m}^{3} / \mathrm{s}$. Hydrographs for these floods were constructed from the reconstruction of historical floods.

\section{Practical safety implications}

In traditional statistical terms (compare Table 1) the probability of exceedance for the design basis river flow is in the range between $10^{-5}$ and $10^{-8}$. This indicates that traditional statistical analysis may lead to an underestimation of the flood hazard and therefore also of flood risk. The results obtained were also compared with a physics-based deterministic flood hazard assessment. The latter was based on the development of scenarios taking into account combinations of unfavourable precipitation conditions with unfavourable retention and outflow conditions from the Swiss lakes. The scenarios were evaluated using a combination of simulation tools and expert judgement. As a result a design basis flood of $1900 \mathrm{~m}^{3} / \mathrm{s}$ was suggested, while the river flow corresponding to maximum credible flood conditions (MCF) was found to make up $2500 \mathrm{~m}^{3} / \mathrm{s}$. The procedure developed for the design of critical infrastructures delivers similar results without the need to refer to subjective expert judgement associated with the development of scenario floods. 


\section{References}

[1] IAEA, "The Fukushima Daiichi Accident, Report by the Director general," IAEA, Vienna, 2015.

[2] WENRA, "Guidance Document Issue T: Natural Hazards Head Document. Guidance for the WENRA safety Reference levels for natural hazards introduced as lesson learned from TEPCO Fukushima dai-ichi accident,", 2015.

[3] INSAG, "A Framework for an Risk-Informed Decision Making Process. A Report by the International Nuclear Safety Group, INSAG -25," IAEA, Vienna, 2011.

[4] Deutsche Vereinigung für Wasserwirtschaft, Abwasser und Abfall e.v. (DWA), "Merkblatt DWA-M 552. Ermittlung von Hochwasserwahrscheinlichkeiten," DWA, Hennef, 2012.

[5] KTA, "KTA 2207. Flood Protection for Nuclear Power Plants.," KTA, Salzgitter, 2009.

[6] J.-U. Klügel, "Probabilistic safety analysis of external floods - method and application," Kerntechnik 78 (2), pp. 127-132, 2013.

[7] N. Taleb, The Black Swan, the impact of the highly improbable, Random House, 2007.

[8] J.-U. Klügel, "Consideration of "Black Swan" Events in the Seismic Safety Review and the Seismic Upgrade Programme of Existing Nuclear Power Plants - the NPP Goesgen Example," in Post-SmiRT23 Seminar, Istanbul, 2015.

[9] J.-U. Klügel, "Risk and Hazard Assessment of Extreme Natural Events for Critical Infrastructures," in Risk Analysis 2016, Crete, Greece, May 25-27, 2016.

[10] P. Embrechts, C. Klüppelberg and T. Mikosch, Modelling Extremal Events for Insurance and Finance, Berlin, Heidelberg, New York: Springer, 1997.

[11] G. Woo, The Mathematics of Natural Catastrophes, London: Imperial College Press, 1999.

[12] NRC, Handbook of Parameter Estimation for Probabilistic Risk Assessment, NUREG/CR-6823, Washington: U.S. Nuclear Regulatory Commission, 2003.

[13] V.B. Nevzorov, "Records: Mathematical Theory, Translations of Mathematical Monographs," American Mathematical Society, Washington, 2001.

[14] R.M. Cook and D. Nieboor, "Heavy-Tailed Distributions: Data, Diagnostics and New Developments, RFF DP 11-19," Resources for the Future, Washington, 2011.

[15] S.F. Resnick, Heavy-Tail Phenomena. Probabilistic and Statistical Modeling., Berlin, Heidelberg, New York: Springer, 2009.

[16] D. Vose, Risk Analysis: A Quantitative Guide, Third Edition, Chichester: John Wiley \& Sons, 2008. 